\title{
Digital single-operator pancreatoscopy for evaluation of intraductal papillary mucinous neoplasm
}

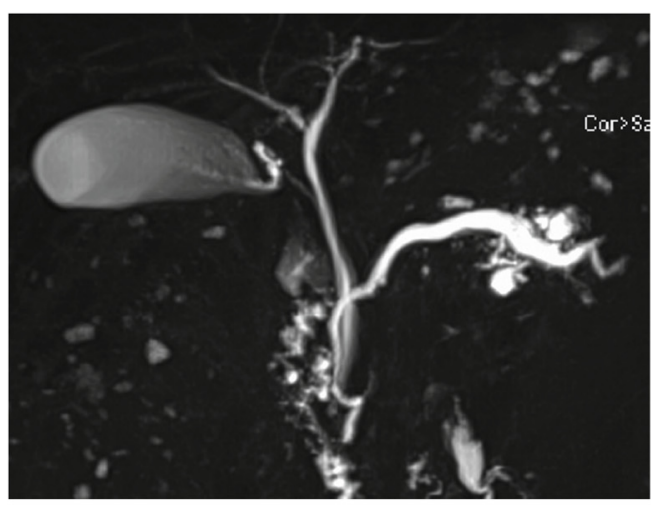

A

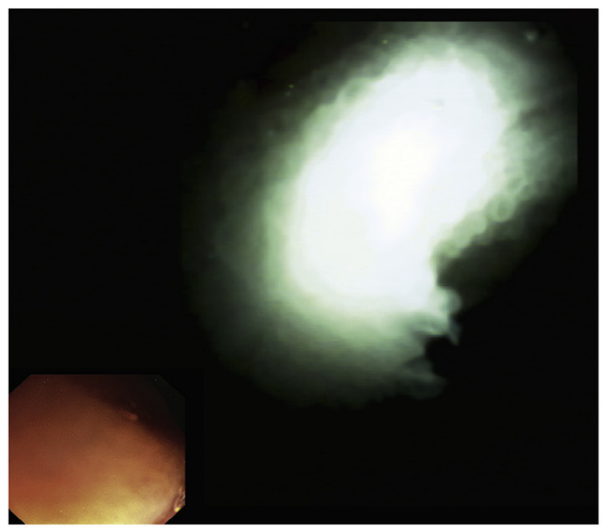

C

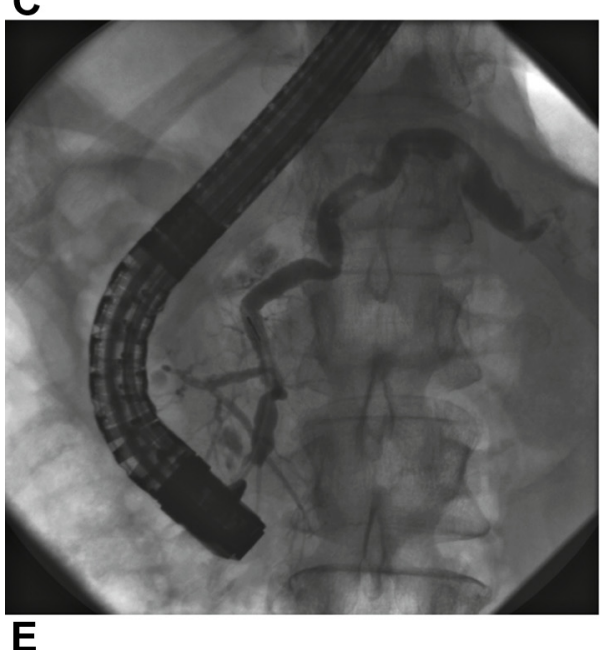

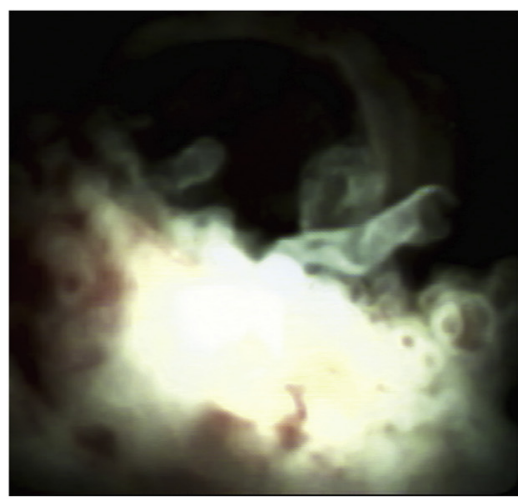

B

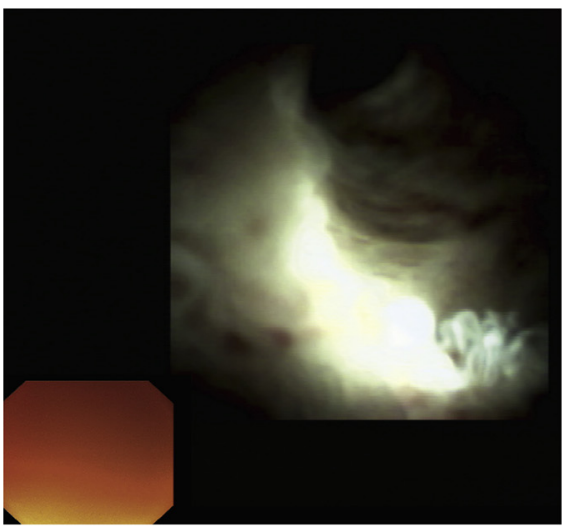

D

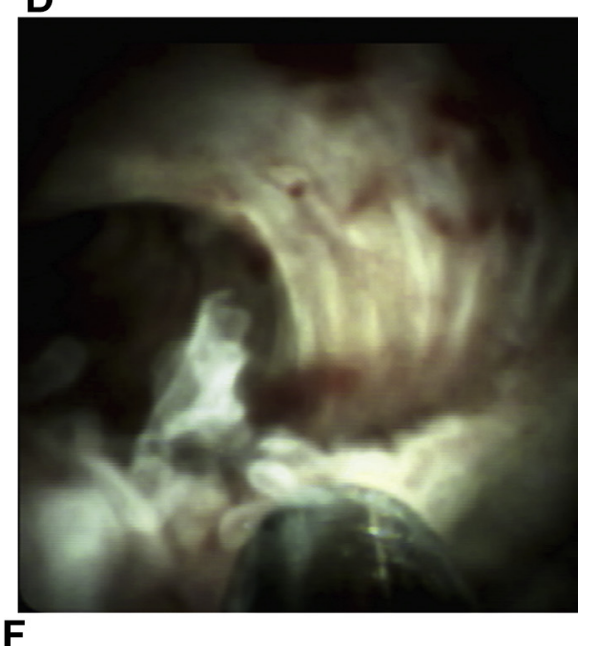

Figure 1. A, Magnetic resonance image showing 2 cystic lesions in the pancreas and mild dilatation of the main pancreatic duct. B, Fish-egg-like lesions in the main pancreatic duct. C, Very small lesions at the bifurcation of a branch duct. D, Normal pancreatic main duct with margin of papillary lesions in front. E, Endoscopic retrograde pancreatography showing mild dilatation of the pancreatic duct. The cystic lesions cannot be demonstrated. F, Neoplastic mucosa with biopsy forceps in front.

Written transcript of the video audio is available online at www.VideoGIE.org. 
Although main-duct intraductal papillary mucinous neoplasm (IPMN) and mixed-type IPMN are frequently associated with malignancy and usually lead to surgery, branch-duct IPMNs biologically behave in a more indolent manner. Prediction of malignancy is a crucial point to distinguish between surgical therapy and conservative management in this heterogenous group of cystic neoplasia. We report on a 58-year-old woman with pancreatic carcinoma in her family history who presented with 2 cystic lesions identified by magnetic resonance imaging for diagnostic workup (Fig. 1A). In an EUS scan, mild dilatation of the main pancreatic duct measuring $3.5 \mathrm{~mm}$ in the head and $4.5 \mathrm{~mm}$ in the body was identified (Video 1, available online at www.VideoGIE.org). Two cystic structures between 10 and $13 \mathrm{~mm}$ with small wall standing nodules and wall thickening were detected. The connecting branch ducts leading from the cystic lesions to the main pancreatic duct could be clearly identified. To discriminate between debris and neoplasia, contrastenhanced EUS was performed and showed no perfusion in the suspected areas. Because involvement of the main duct could not be excluded, pancreatoscopy with the SpyGlass DS system (Boston Scientific, Marlborough, Mass) was performed for further evaluation. Through ERCP (Fig. 1E), the pancreatic duct was cannulated with a 0.035-inch guidewire (Jagwire, Boston Scientific), and sphincterotomy was performed (Cotton Sphincterotome, Cook Medical, Bloomington, Ind). Subsequently, the SpyGlass DS catheter was advanced into the pancreatic duct. In the area of the cystic structures, multiple fishegg-like lesions could be identified in the main pancreatic duct (Fig. 1B-D). Biopsy specimens were taken under direct endoscopic visualization (Fig. 1F) and showed papillary clusters of bland-appearing columnar cells with abundant intracytoplasmic mucin. The mucin filled the entire cytoplasm and displaced the nucleus to the periphery or base of the cell. In consequence of the verification of main-duct involvement, surgical resection was recommended. The tail and part of the body of the organ were resected, and a diagnosis of mixed-type IPMN with lowgrade dysplasia was confirmed by pathologic examination. In summary, intraductal pancreatoscopy and biopsy can provide important information in IPMN to confirm a diagnosis.

\section{DISCLOSURE}

All authors disclosed no financial relationships relevant to this publication.

David Albers, MD, Dani Dakkak, MD, Department of Gastroenterology, Elisabeth-Krankenhaus Essen, Teaching Hospital of the University of Duisburg-Essen, Essen, Germany, William Sterlacci, MD, PhD, Michael Vieth, MD, PhD, Department of Pathology, Klinikum Bayreuth, Bayreuth, Germany, Teaching Hospital of the University of Erlangen, Erlangen, Germany, Brigitte Schumacher, MD, PhD, Department of Gastroenterology, Elisabeth-Krankenhaus Essen, Teaching Hospital of the University of Duisburg-Essen, Essen, Germany

Copyright $@ 2016$ The Authors. Published by Elsevier, Inc. on behalf of the American Society for Gastrointestinal Endoscopy. This is an open access article under the CC BY-NC-ND license (http://creativecommons.org/ licenses/by-nc-nd/4.0/).

http://dx.doi.org/10.1016/j.vgie.2016.10.007 